\title{
Valoración de impactos ecológicos por minería de oro en río Guabas, Valle del Cauca, Colombia
}

\section{Assessment of ecological impacts by mining of gold in Guabas River, Valle del Cauca, Colombia}

\author{
Avaliação dos impactos ecológicos de mineração \\ de ouro do rio Guabas, Valle del Cauca, Colômbia
}

\author{
Diego Esteban Gamboa García \\ Ingeniero Agrícola
}

Facultad de Ingeniería y Administración. Grupo Ecología y Contaminación Acuática. Universidad Nacional de Colombia Sede Palmira. Colombia.

degamboag@unal.edu.co

\section{Resumen}

La minería de oro es una actividad que se ha desarrollado por siglos en diferentes territorios en el planeta. Sin embargo, la minería ha generado impactos ambientales afectando a la sociedad, debido a que comprometen la cantidad y calidad de los servicios ecosistémicos y en consecuencia, la calidad de vida de la población mundial. Para medir el nivel de afectación sobre los componentes del ambiente, se revisaron los métodos clásicos para Estudios de Impacto Ambiental (EIA) para proyectos mineros y se tomó como base la matriz de Leopold desarrollada por el Servicio Geológico del Departamento del Interior de los Estados Unidos, para valorizar los impactos de actividades mineras. En esta investigación se revisaron investigaciones publicadas con las palabras clave "matriz cualitativa" y "minería de oro", tanto en inglés como en español, desde el año 2000 al 2011, en proyectos mineros realizados en países en vía de desarrollo. Posteriormente, se extrajeron y sistematizaron, las metodologías clásicas y sus variaciones y los componentes ecológicos y actividades del proyecto comunes en los estudios. Las metodologías usadas fueron cualitativas. Adicionalmente, se desarrolló la metodología Valor del Índice Ambiental (VIA) para valorizar los impactos ecológicos de la minería de oro en la cuenca del río Guabas, Valle del Cauca, Colombia. Se encontró que el impacto promedio de la actividad minera desarrollada en el río Guabas fue considerado severo, a diferencia del impacto global revisado en la literatura, que fue considerado como moderado, en la mayoría de los casos.

Palabras clave: Estudios Impacto Ambiental, metodologías cualitativas, extracción minera

\section{Abstract \\ Gold mining is an activity that has been developed for centuries in different territories on the planet. However, mining has generated environmental impacts affecting society, because they affect the quantity and quality of ecosystem services and therefore the quality of life of the world population. To measure the degree of affectation on the com- ponents of the environment, the classical methods for studies of environmental impact (EIA) for mi- ning projects were reviewed the matrix of Leopold developed by the Geological Service from the De- partment of the Interior of the United States, was taken as a basis, to value the impacts of mining ac- tivities. In this research published articles with the}


keywords "Matrix qualitative" and "mining of gold", both English and Spanish, from 2000 to 2011, in mining projects carried out in developing countrie were reviewed. Subsequently classical methodologies and their variations and ecological components and common project activities in the studie were extracted and systematized. The methodologies used were qualitative. Additionally, the value of the environmental index (VIA) methodology was developed to value the ecological impacts of gold mining in the basin of the Guabas River, Valle del Cauca, Colombia. It was found that the average impact of mining activity in the Guabas River was considered severe, in comparison to the overall impact reviewed in the literature, which were considered as moderate, in most cases.

Key-words: Environmental Impact Assessments, qualitative methodologies, mining extraction

\section{Resumo}

Mineração de ouro é uma atividade que tem sido desenvolvida ao longo dos séculos em diferentes territórios do planeta. No entanto, a mineração tem gerado impactos ambientais que afectam a sociedade, porque eles comprometem a quantidade ea qualidade dos serviços dos ecossistemas e, consequentemente, a qualidade de vida da população mundial. Para medir o nível de impacto sobre componentes do ambiente, os métodos tradicionais para Avaliações de Impacto Ambiental (EIA) foram revistos para projetos de mineração e foi baseado na matriz de Leopold desenvolvido pelo Serviço Geológico do Departamento do Interior dos Estados Unidos, para valorizar os impactos das atividades de mineração. Nesta pesquisa, têm sido revisadas as publicações com as palavras chave "matriz qualitativa" e "mineração de ouro" em Inglês e Espanhol, de 2000 a 2011, em projetos de mineração de países em desenvolvimento foram revistas publicações. Estas foram extraídas para ser sistematizadas suas metodologías clássicas e suas variações e componentes ecológicos e actividades do projeto, comum nos estudos. As metodologias utilizadas foram qualitativas. Além disso, a metodologia Índice de Valor Ambiental (VIA) foi desenvolvido para melhorar os impactos ecológicos da mineração de ouro na bacia do rio Guabas, Valle del Cauca, Colômbia. Verificou-se que o impacto médio da atividade de mineração no rio Guabas foi considerada grave, ao contrário do impacto global revisto na literatura, onde foi considerada moderada, na maioria dos casos .

Palavras-chave: Estudos de Impacto Ambiental, metodologias qualitativas, mineração

\section{Introducción}

Los conflictos por la ejecución de los proyectos mineros son principalmente por el territorio (Urkidi, 2010) lo cual ha generado puntos de vista opuestos entre las comunidades locales, los gobernantes, los empresarios y los profesionales ambientales. Por tanto, el objeto de esta investigación es identificar y resumir los impactos ecológicos asociados a la minería de oro y la valoración que tienen en los países en desarrollo. Por otro lado, se pretende aplicar una metodología cualitativa rápida y sencilla de Estudios de Impacto Ambiental (EIA) para las actividades mineras en la cuenca del Río Guabas, Valle del Cauca, Colombia. Finalmente, se realiza una comparación entre la cuenca del río Guabas y la valoración de impactos de minería a escala de los países en desarrollo.

En la minería de metales, Swart \& Dewulf (2013) argumentan que la demanda de cobre, zinc, plomo, níquel, molibdeno, oro, plata, paladio y platino, ha aumentado por el desarrollo tecnológico que precisa de estos metales específicos, aunque, su extracción implica grandes volúmenes de roca mineral en todos sus estados. Respecto a esto, el oro ocupa el primer lugar, existiendo un incremento anual de $15 \mathrm{t}$ de material minero por cada $\mathrm{kg}$ de 
oro obtenido. Sin embargo, el impacto positivo de la minería de metales se relaciona con el factor humano, pues se argumenta la generación de empleo, riqueza y relaciones interindustriales.

La percepción positiva de la minería en la generación de riqueza y empleo para la sociedad es una hipótesis lógica, sin embargo, Pegg (2006), reportó que los países más dependientes de la minería en la contribución a su producto interno tienen menor crecimiento económico y mayor inequidad social. Aun así, el capital trasnacional es una presión externa sobre los depósitos de oro, donde la mayor inversión para la exploración mundial de este metal se ha centrado en los países en desarrollo (Urkidi, 2010). Respecto a esta categoría socioeconómica, Latinoamérica es la región más importante en la exploración mundial de yacimientos de oro, donde en la década de 1995 a 2004 , se realizaron en promedio el $26 \%$ de estas actividades de exploración (USGS, 2015). Aunque todos los sitios de exploración no se convierten en sitios de extracción de oro, una vez determinado el yacimiento del mineral, se desarrollan una serie de actividades que generan impactos sobre algunos componentes del ambiente. En consecuencia, los países en desarrollo han venido aplicando diferentes metodologías de EIA con el objetivo de definir el alcance, identificación, predicción y evaluación de los impactos de los proyectos mineros, para soportar la toma de decisiones (Cuentas, 2009). El alcance, la identificación, la predicción y la evaluación de los impactos de los proyectos de minería de oro dependen tanto de la escala de extracción y el tipo de yacimiento, como del grado de tecnificación.

En un EIA realizado en la Guyana venezolana, Lozada \& Arends, (2000) identificaron las fases de la minería de oro, su escala y sus impactos. Allí se explican de la siguiente manera:

1. Prospección: se determina la presencia del metal y se delinean las áreas de mayor potencial. Se utilizan métodos de sensibilidad magnética donde se generan mapas de contraste.
2. Exploración: se cuantifica el volumen de yacimientos: $g$ de oro/t de material que lo contiene.

3. Explotación: según la productividad de la mina, así mismo se considera la siguiente clasificación de escala: artesanal (menos de $5 \mathrm{~kg} /$ año), pequeña (5 a $50 \mathrm{~kg} / \mathrm{año}$ ), mediana (50 a $1000 \mathrm{~kg} / \mathrm{año}$ ), grande (1 a 10 t/año) y junior (10 a 50 t/año).

4. Procesamiento: consiste en separar el oro del material estéril. Si el oro está en roca, ésta debe ser molida, ocasionando polvo y material particulado, el cual puede afectar las vías respiratorias de los animales terrestres. Se puede procesar con amalgama de mercurio o plantas de cianuro. En el primer proceso, la superficie del oro es mojada y absorbida por el mercurio, siendo un proceso de mojado y no de disolución. El oro se separa de la amalgama por destilación, para ello, los mineros colocan la mezcla sobre una placa metálica y la queman con un soplete. Los impactos ecológicos de esta práctica se relacionan con la liberación de $\mathrm{Hg}$ al ambiente, con consecuencias que se mencionarán más adelante. Mientras que las pocetas de cianuro, donde se recupera el 95\% del oro contenido, funcionan de la siguiente manera: Se agrega $\mathrm{NaCN}$ a un lodo aurífero para producir $\mathrm{NaAu}(\mathrm{CN})_{2}$ que en presencia de $\mathrm{Zn}$ genera $\mathrm{Na}_{2} \mathrm{Zn}(\mathrm{CN})_{4}$ y oro libre $(\mathrm{Au})$. Existe una ventaja de esta práctica y es que el cianuro puede ser reciclado hasta en un $90 \%$. Sin embargo, durante las lluvias estas pocetas se desbordan, y el cianuro se mueve hasta los cauces, disminuyendo el $\mathrm{pH}$ del agua por su carácter ácido y perjudicando la vida acuática.

En los ecosistemas, los efectos del mercurio pueden ser rápidos o crónicos. Esto debido a la versatilidad del $\mathrm{Hg}$ en el ambiente (Harris, Pickering \& George, 2003) y a que se puede bioacumular y biomagnificar en la red trófica (Cogua, CamposCampos \& Duque, 2012). En el contexto global, la mirada investigativa sobre los impactos ecológicos por la liberación de Hg, se despertó por la 
epidemia en Minamata, Japón; en esta catástrofe del año 1956, la población ingirió sin conocimiento peces contaminados con mercurio en su dieta diaria, lo que generó efectos de toxicidad crónica y letal, tales como trastornos sensoriales, contracción concéntrica del campo visual, casos de ataxia y problemas del habla (Marrugo, Ruiz \& Diez, 2013) y se diagnosticaron consecuencias en el funcionamiento cerebral del feto (Corredor, 2013).

En el caso de los impactos sobre la fauna en peces, por ejemplo, se cuenta con un estudio con trucha moteada (Cynoscion nebulosus) que demostró que el mercurio causa inflamación intestinal, degeneración tubular y necrosis renal (Adams et al., 2010). Mientras que en aves, entre los efectos por toxicidad con mercurio, se encuentra la afección al sistema nervioso, circulatorio y endocrino, generando un riesgo en la viabilidad de la población (Bowerman, Evans, Giesy \& Postupalsky, 1994; Burger \& Gochfeld, 2004; Cristol, Mojica, Varian-Ramos, \& Watts, 2012; Burger, Jehl y Gochfeld, 2013; Burger, 2013) y un amplio rango de efectos en la reproducción, incluyendo bajo peso del huevo, malformaciones en el embrión, detrimento y alteraciones en el crecimiento, menos sobrevivencia de los polluelos, así como daños nerviosos y esterilidad (Burger \& Gochfeld, 2004).

La minería de oro poco tecnificada durante la fase de preparación, tiene la amalgamación del oro con mercurio, como una de las actividades que genera mayores impactos sobre los componentes ambientales, en lo que coinciden diferentes EIA. La situación se agudiza, debido a que la minería de oro es una de las principales fuentes de $\mathrm{Hg}$ en el ambiente (Olivero, Johnson \& ArgueIlo, 2002; Marrugo, Lanz \& Benítez, 2007), pues del total del mercurio usado para separar el oro del resto de material, una parte se pierde por vertimiento y otra parte en forma de vapor durante la quema de la amalgama. Respecto a esto, Ogola, Mitullah \& Omulo (2002) encontraron que se liberan al ambiente 1.3 partes de $\mathrm{Hg}$ por cada parte de oro producido.

\section{Metodología}

\section{Valoración cualitativa promedio de los impactos por minería de oro en los países en desarrollo}

Se consultaron en bases de datos investigaciones publicadas, con las palabras clave "Matriz cualitativa" y "Minería de oro", tanto en inglés como en español, en países en vía de desarrollo, desde el año 2000 hasta el 2011. Posteriormente, se extrajeron y sistematizaron, tanto las metodologías clásicas y sus variaciones, como los componentes ambientales y las actividades del proyecto, comunes en la mayoría de los estudios. Los componentes ambientales que se consideraron fueron, Suelo, Aire, Agua, Vegetación y Fauna. Cada componente tuvo una valoración general extraída del estudio (Tabla 1) y se realizó una valoración total de los componentes impactados en los países en desarrollo, según la bibliografía consultada. Además, se reportaron los estudios en los que se identificaron las actividades de importancia relacionadas con el uso de $\mathrm{Hg}$ en la extracción del oro. También se analizó si los estudios de caso donde se desarrollaron los EIA, tenían impactos previos y cuál era su naturaleza.

Tabla 1. Valoración general de los impactos sobre el componente ambiental, extraída de cada estudio de caso

\begin{tabular}{cc}
\hline $\begin{array}{c}\text { Valoración } \\
\text { general }\end{array}$ & $\begin{array}{c}\text { Significado extraído } \\
\text { del estudio de caso }\end{array}$ \\
\hline
\end{tabular}

Se identificó el impacto sobre el componente y su valoración fue negativa

Se identificó el impacto sobre el componente y su valoración fue positiva

0

Se identificó el impacto sobre el componente y su valoración fue tanto positiva como negativa

No se identificó el impacto sobre el componente 
Se revisaron siete Estudios de Impacto Ambien- Guyana-Venezuela, Migori-Kenya, Lima-Perú, tal (EIA) sobre minería de oro del año 2000 a Puno-Perú, Antioquia-Colombia (2 casos) y Ama2011 que comprenden las siguientes regiones: zonas-Brasil (Tabla 2).

Tabla 2. Identificación de los componentes ecológicos y valoración de los impactos por actividades de minería de oro en cada estudio de caso.

\begin{tabular}{|c|c|c|c|c|c|c|c|c|c|c|c|}
\hline Cita & IP & País & Ciudad & Año & Metodología & s & $\mathbf{A i}$ & A & V & $\mathbf{F}$ & $\mathrm{Hg}$ \\
\hline $\begin{array}{l}\text { (Lozada } \\
\& \text { Arends, } \\
\text { 2000) }\end{array}$ & $\mathrm{X}$ & Venezuela & Guyana & 2000 & Matriz de Leopold & -1 & -1 & -1 & -1 & -1 & -1 \\
\hline $\begin{array}{l}\text { (Ogola et } \\
\text { al., 2002) }\end{array}$ & $x$ & Kenya & Migori & 2002 & $\begin{array}{l}\text { Lista chequeo, entrevistas } \\
\text { informales y muestreos } \\
\text { cuantitativos }\end{array}$ & -1 & -1 & -1 & -1 & -1 & -1 \\
\hline $\begin{array}{l}\text { (Gallar- } \\
\text { day, 2006) }\end{array}$ & $\mathrm{X}$ & Perú & Lima & 2006 & Matriz de Leopold & 0 & -1 & & -1 & -1 & \\
\hline $\begin{array}{l}\text { (Molina et } \\
\text { al, 2008) }\end{array}$ & $\mathrm{X}$ & Colombia & $\begin{array}{l}\text { El } \\
\text { Bagre, } \\
\text { Antio- } \\
\text { quia }\end{array}$ & 2008 & $\begin{array}{l}\text { Medición del impacto económi- } \\
\text { co involucra tanto a la empre- } \\
\text { sa, como a la comunidad y al } \\
\text { Estado }\end{array}$ & & & & & & \\
\hline $\begin{array}{l}\text { (Moreno, } \\
\text { 2013) }\end{array}$ & $\mathrm{X}$ & Colombia & $\begin{array}{l}\text { El } \\
\text { Bagre, } \\
\text { Antio- } \\
\text { quia }\end{array}$ & 2008 & $\begin{array}{l}\text { Se tomó unos grupos de } 25 \\
\text { personas de ocupación minera } \\
\text { y se sometieron a una batería } \\
\text { de pruebas de personalidad y } \\
\text { comportamiento }\end{array}$ & & & & & & -1 \\
\hline $\begin{array}{l}\text { (Sousa et } \\
\text { al., 2011) }\end{array}$ & $\mathrm{X}$ & Brasil & $\begin{array}{l}\text { Amazo- } \\
\text { nas }\end{array}$ & 2011 & $\begin{array}{l}\text { Matriz de Leopold modificada. } \\
\text { Enfoque Heurístico. Considera } \\
\text { los impactos medibles directa- } \\
\text { mente, y la percepción de los } \\
\text { interesados de tales impactos }\end{array}$ & -1 & & -1 & -1 & & -1 \\
\hline $\begin{array}{l}\text { (Cuentas, } \\
\text { 2009) }\end{array}$ & $\mathrm{X}$ & Perú & Puno & 2009 & $\begin{array}{l}\text { Esta modalidad de minería } \\
\text { opera con una planta que } \\
\text { vincula a } 84 \text { trabajadores y } \\
\text { recibe el trabajo de } 400 \text { minero } \\
\text { artesanales. Para el Estudio en } \\
\text { cuestión sólo se considera la } \\
\text { etapa de exploración. Método } \\
\text { de Criterios Relevantes Integra- } \\
\text { dos: Propone la elaboración del } \\
\text { índice de VIA (Valor Impacto } \\
\text { Ambiental). El método RIAM } \\
\text { (Evaluación Rápida de Impac- } \\
\text { tos Ambientales), clasifica a los } \\
\text { componentes ambientales en } 4 \\
\text { categorías } \\
\text { ambientales: ambiente físico, } \\
\text { ambiente biológico, ambiente } \\
\text { social cultural y ambiente } \\
\text { económico. Método Vicente } \\
\text { Conesa }\end{array}$ & -1 & & -1 & -1 & & -1 \\
\hline
\end{tabular}

IP: Impactos previos. Componentes: S (Suelo), Ai (Aire), A (Agua), V (Vegetación), F (Fauna) y Hg (actividades con manipulación y liberación de mercurio). 


\section{Zona de estudio}

EL río Guabas hace parte de la cuenca SonsoGuabas, zona de reserva forestal protectora, que se encuentra en el departamento del Valle del Cauca, en el flanco occidental de la Cordillera Central de Colombia. Allí se ubica un complejo minero Ilamado la Victoria, donde según el Programa Agropecuario Municipal (Alcaldía municipal de Ginebra, 2015), se practica la minería a escala artesanal, realizando labores de acceso subterráneo o a cielo abierto dependiendo de la profundidad a la que se encuentre el mineral.

\section{Panel de expertos}

El panel de expertos realizó una visita a la mina La Suiza, en el complejo minero la Victoria, donde se encontró una mina en teoría inactiva, debido a que el molino, y los pizones estaban en mal estado. Se tomaron fotografías y se realizaron entrevistas a diferentes actores sociales con el objetivo de ampliar la percepción sobre los impactos percibidos por la actividad en los actores sociales. En adición, se revisó literatura de las diferentes organizaciones gubernamentales que han traba- jado sobre el estado e impacto de las actividades mineras en la zona.

Se realizó una matriz de Criterios Relevantes Integrados (CRI) (Buroz, 1994), determinando la relación causa-efecto ubicando los componentes ecológicos que pueden ser afectados en las columnas, y las fases de los proyectos mineros, aspectos y sub-aspectos en las filas; resultando en los impactos del proyecto al ecosistema. A través de la interacción o no, de las actividades mineras desarrolladas con los componentes ecológicos, se identificaron y valorizaron los impactos ecológicos y se definieron los impactos significativos, llegando hasta la valoración de SEVERIDAD, a través de la secuencia lógica mostrada en las Tablas 3 y 4.

En cada celda de la matriz, se le da un valor a Intensidad, Extensión y Duración. Con estos valores se calcula la Magnitud (M) del Impacto (ecuación 1). Posteriormente se asigna la variable Reversibilidad e Incidencia a cada interacción y se obtiene Valor de Índice Ambiental (VIA) (ecuación 2). Luego la severidad se calcula mediante el producto entre VIA y M (ecuación 3).

Tabla 3. Criterios para valorar impactos ecológicos

\begin{tabular}{|c|c|c|c|c|}
\hline PARÁMETRO & CRITERIO & \multicolumn{2}{|c|}{ ESCALA } & VALOR \\
\hline \multirow{4}{*}{$\begin{array}{l}\text { Intensidad } \\
\text { del impacto (I) }\end{array}$} & $\begin{array}{l}\text { Se refiere al grado con el que un impacto altera a un determinado } \\
\text { elemento del ambiente, por tanto está en relación con la fragilidad y }\end{array}$ & \multicolumn{2}{|c|}{ Alto } & $7-9$ \\
\hline & sensibilidad de dicho elemento, puede ser alto, medio o bajo. El valor & \multirow{2}{*}{\multicolumn{2}{|c|}{ Medio }} & $4-6$ \\
\hline & sufrido. Esta calificación de carácter subjetivo estable la predicción & & & \\
\hline & del cambio neto entre las condiciones, con y sin proyecto. & \multicolumn{2}{|c|}{ Bajo } & $1-3$ \\
\hline \multirow{3}{*}{$\begin{array}{l}\text { Extensión o } \\
\text { influencia } \\
\text { espacial (E) }\end{array}$} & Determina el área geográfica de influencia teórica que será afecta- & \multicolumn{2}{|c|}{ Regional } & 10 \\
\hline & da por un impacto en relación con el entorno del proyecto (porcen- & \multicolumn{2}{|c|}{ Local } & 5 \\
\hline & efecto), pudiendo esta ser puntual, local, regional. & \multicolumn{2}{|c|}{ Puntual } & \\
\hline \multirow{3}{*}{ Duración (D) } & \multirow{3}{*}{$\begin{array}{l}\text { Se refiere al tiempo que supuestamente permanecería el efecto, } \\
\text { desde su aparición, y a partir del cual el factor afectado retornaría } \\
\text { a las condiciones iniciales, previo a la acción de medios naturales } \\
\text { o mediante la introducción de medidas correctoras. La duración es } \\
\text { independiente de la reversibilidad }\end{array}$} & (>10 años) & Largo & 10 \\
\hline & & (5-10 años) & Mediano & 5 \\
\hline & & (0-5 años) & Corto & 2 \\
\hline
\end{tabular}

Fuente: Buroz (1994). 
Cálculo de la Magnitud del Impacto

$\mathrm{M}=\left(I^{*} \mathrm{~W}\right)+\left(\mathrm{E}^{*} \mathrm{WE}\right)+\left(\mathrm{D}^{*} \mathrm{WD}\right)$,

Donde,

M=Magnitud

I=Intensidad

$\mathrm{W}=$ peso específico de Intensidad, para este caso (0.4)

$\mathrm{E}=$ Extensión

WE=peso específico de Extensión (0.3)

$\mathrm{D}=$ Duración

WD=Peso específico duración (0.3)

Tabla 4. Criterios para valorar impactos ecológicos

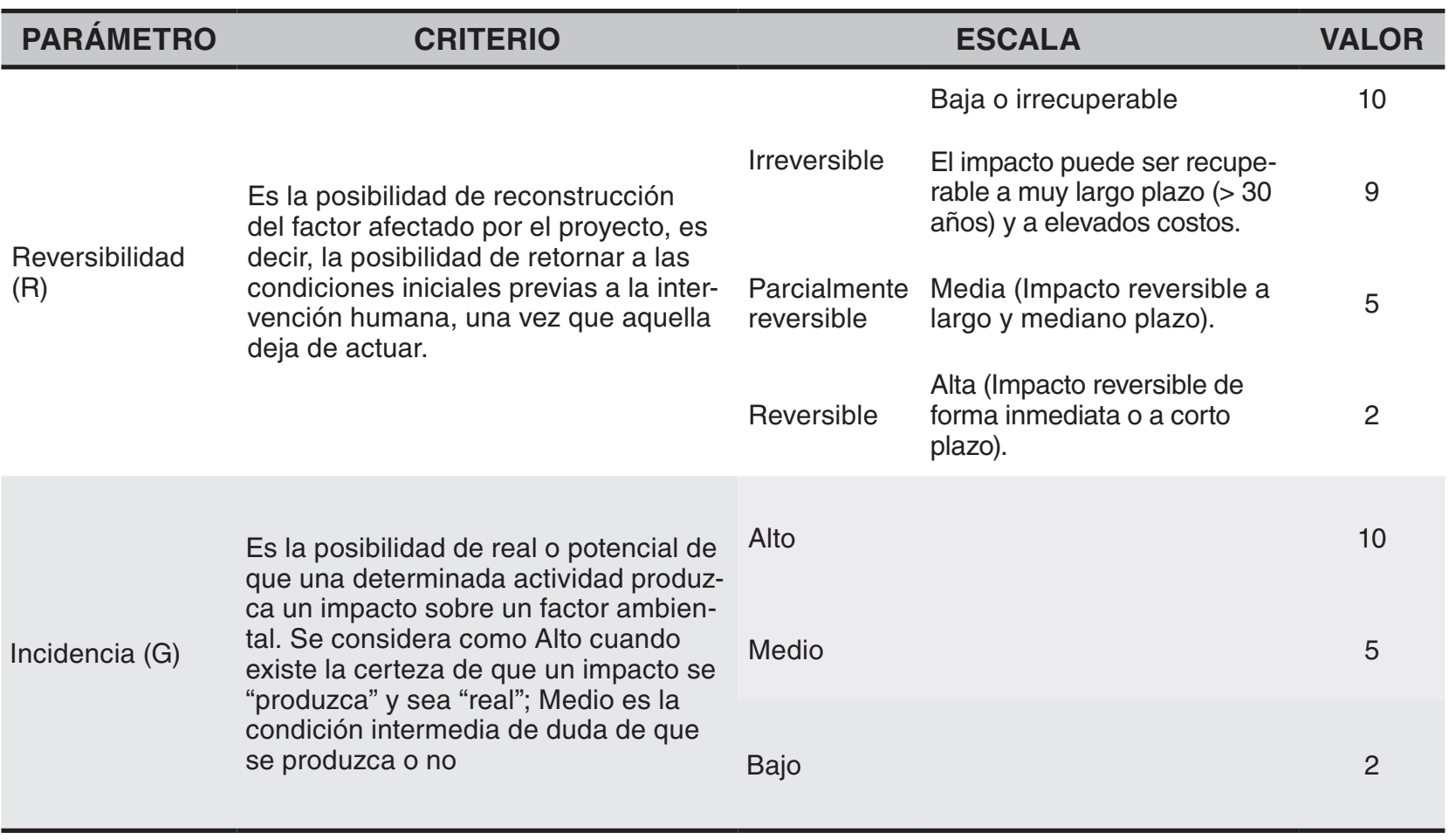

Fuente (Buroz, 1994)

Fórmula de Valor de Índice Ambiental (VIA)

$\mathrm{VIA}=(\mathrm{RiXR} \times \mathrm{GiXG} \times \mathrm{MXM})$,

Donde,

$\mathrm{VIA}=$ Valor de Indice Ambiental

$\mathrm{XM}=$ peso de la variable magnitud (0.4)

$X G=$ peso de la variable Incidencia (0.2)

$\mathrm{XR}=$ peso de la variable reversibilidad (0.4)
Fórmula Severidad

$\mathrm{S}=\mathrm{VIA} \times \mathrm{M}$,

(3)

Donde,

$\mathrm{S}=$ Severidad

VIA=Valor de Indice Ambiental

$\mathrm{M}=$ Magnitud 
Por último se realizó una comparación entre los impactos significativos identificados en las actividades mineras en la cuenca del río Guabas y los impactos identificados en la literatura consultada.

\section{Resultados y discusión}

Se revisaron siete investigaciones, donde se desarrollaron EIA por actividades mineras desde el año 2000 a 2011, y comprenden las siguientes regiones: Guyana-Venezuela, Migori-Kenya, LimaPerú, Puno-Perú, Antioquia-Colombia (2 casos) y Amazonas-Brasil. Las metodologías usadas fueron cualitativas, diferentes entre sí. A pesar de las distancias geográficas y las posibles diferencias en el ambiente biofísico entre los sitios, la valoración total del impacto de los proyectos mineros en cada sitio fue moderado. Sólo en un estudio, los componentes suelo y vegetación llegaron a ser impactados a nivel severo.

En las actividades mineras de la cuenca del río Guabas, la fase de trituración y pulverización, en su aspecto molino y pizón, y su sub-aspecto consumo de energía, no tuvieron impacto en el ecosistema, como tampoco la fase de procesamiento en su aspecto mesa concentradora y uso de sustancias peligrosas, y su sub-aspecto consumo de agua (Tabla 5).

Tabla 5. Matriz de Severidad de Impactos: minería de oro en río Guabas

\begin{tabular}{|c|c|c|c|c|c|c|c|c|c|c|c|}
\hline & & MATRIZ DE & SEVERII & DAD DE IM & РАCTO & :MINER & IA DE O & O EN G & JABAS & & \\
\hline \multirow[b]{4}{*}{$\begin{array}{l}\text { ACTI- } \\
\text { VIDAD }\end{array}$} & \multirow[b]{4}{*}{$\begin{array}{l}\text { SUBAC- } \\
\text { TIVIDAD }\end{array}$} & \multicolumn{10}{|c|}{ COMPONENTES ECOLÓGICAS E IMPACTOS } \\
\hline & & \multicolumn{6}{|c|}{ MEDIO FISICO } & \multicolumn{4}{|c|}{ MEDIO BIOTICO } \\
\hline & & \multicolumn{2}{|r|}{ AIRE } & \multicolumn{2}{|c|}{ SUELO } & \multicolumn{2}{|c|}{ AGUA } & \multicolumn{2}{|c|}{ FLORA } & \multicolumn{2}{|c|}{ FAUNA } \\
\hline & & Aspecto & Calidad & $\begin{array}{l}\text { Pérdida } \\
\text { propieda- } \\
\text { des físicas } \\
\text { del suelo }\end{array}$ & $\begin{array}{l}\text { Des- } \\
\text { liza- } \\
\text { miento } \\
\text { suelo }\end{array}$ & $\begin{array}{l}\text { Calidad } \\
\text { físico- } \\
\text { química } \\
\text { del } \\
\text { agua }\end{array}$ & $\begin{array}{l}\text { Reduc- } \\
\text { ción de } \\
\text { oferta } \\
\text { hírdrica }\end{array}$ & $\begin{array}{l}\text { Extin- } \\
\text { ción } \\
\text { espe- } \\
\text { cies } \\
\text { endé- } \\
\text { micas }\end{array}$ & $\begin{array}{l}\text { Toxi- } \\
\text { cidad } \\
\text { flora } \\
\text { acuáti- } \\
\text { ca }\end{array}$ & $\begin{array}{l}\text { Toixi- } \\
\text { cidad } \\
\text { fauna } \\
\text { acuáti- } \\
\text { ca }\end{array}$ & $\begin{array}{l}\text { Desplaza- } \\
\text { miento de } \\
\text { especies }\end{array}$ \\
\hline \multirow{6}{*}{$\begin{array}{l}\text { Exca- } \\
\text { vación }\end{array}$} & $\begin{array}{l}\text { Uso de } \\
\text { Pico y } \\
\text { pala }\end{array}$ & $\begin{array}{l}\text { Emisión } \\
\text { de material } \\
\text { particulado }\end{array}$ & & & & & & & & & \\
\hline & \multirow{3}{*}{$\begin{array}{l}\text { Uso de } \\
\text { Explosi- } \\
\text { vos }\end{array}$} & $\begin{array}{l}\text { Emisión } \\
\text { de material } \\
\text { particulado }\end{array}$ & & & & & & & & & \\
\hline & & $\begin{array}{l}\text { Generación } \\
\text { de Vibración }\end{array}$ & & & & & & & & & \\
\hline & & $\begin{array}{l}\text { Liberación de } \\
\text { sedimentos }\end{array}$ & & & & & & & & & \\
\hline & \multirow{2}{*}{$\begin{array}{l}\text { Abertu- } \\
\text { ra del } \\
\text { Socavón }\end{array}$} & $\begin{array}{l}\text { Aprovechamien- } \\
\text { to de Madera } \\
\text { para postes }\end{array}$ & & & & & & & & & \\
\hline & & $\begin{array}{l}\text { Consumo } \\
\text { de energía }\end{array}$ & & & & & & & & & \\
\hline
\end{tabular}




\section{Continuación Tabla 5}

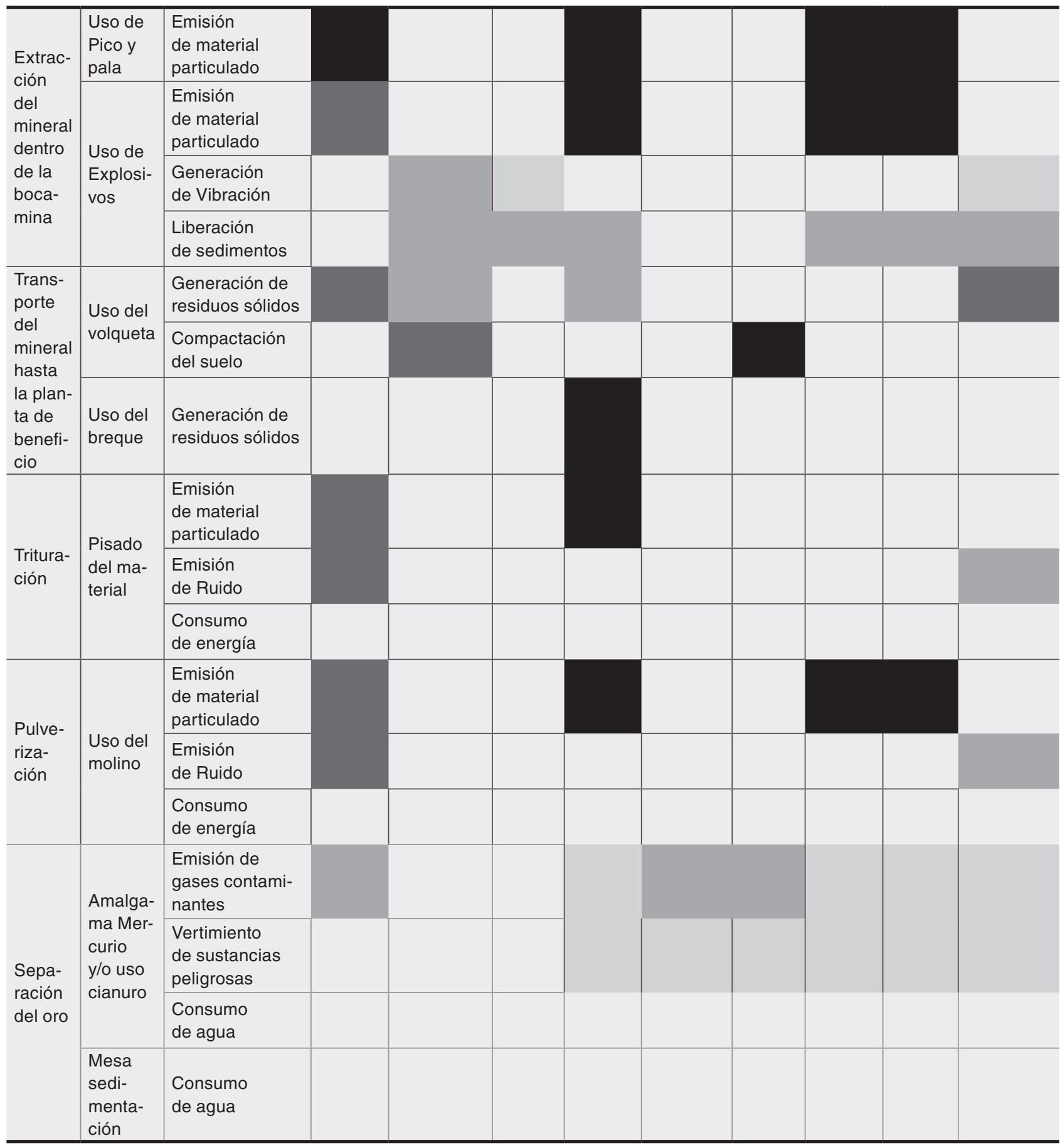

\section{Convenciones}

Leve

Moderado

Severo

Crítico 
Más del $90 \%$ de los componentes ecológicos son afectados de forma severa, lo cual concuerda con la literatura. Los sub-aspectos críticos son el aprovechamiento de recursos maderables, como también la generación de vibración con explosivos, y por último, las emisiones gaseosas y vertimiento de sustancias peligrosas por el uso de mercurio y cianuro.

\section{Conclusiones}

El impacto promedio, o general de la actividad minera desarrollada en el río Guabas fue considerado severo, lo que se diferencia con el impacto global revisado en la literatura, que fueron considerados como moderado en la mayoría de los casos. Mientras que el impacto ecológico sobre los componentes suelo y vegetación concuerdan en su valoración severa.

El análisis de las herramientas académicas permite concluir que los proyectos mineros generan impactos ecológicos predominantemente moderados, lo cual puede explicar la proliferación de propuestas de extracción y la ejecución de políticas mineras, como en el caso de Colombia y la denominada "locomotora minera", donde el gobierno 2010-2014 se ha interesado en participar de las ganancias de la minería, debido a que ha encontrado un crecimiento del producto interno asociado a las actividades extractivas; no obstante, esta fuente de riqueza se está convirtiendo en una nueva fuente de conflictividad social en departamentos como Santander, Tolima y Antioquia.

\section{Agradecimientos}

El autor agradece a la institución Universidad Nacional Sede Palmira, en su programa de posgrado de Maestría en Ingeniería Ambiental de la Facultad de Ingeniería y Administración, que a través del apoyo para la realización de las salidas a la Cuenca Sonso-Guabas permitió la realización de esta investigación. También agradece al profesor Gabriel Fernández, a las ingenieras Liliana León,Viviana Tafur y Luisa Usman, quienes con su experiencia en Evaluación de Impacto Ambiental apoyaron la conceptualización del estudio; sin su conocimiento, experiencia y apoyo no hubiera sido posible la investigación. Finalmente agradece a los miembros del Área protegida Cuenca Sonso-Guabas.

\section{Literatura citada}

1. Alcaldía Municipal de Ginebra. (2015). Programa Agropecuario Municipal - PAM "Unidos por el cambio y el progreso de Ginebra”. Ginebra, Colombia

2. Adams, D. H., Sonne, C., Basu, N., Dietz, R., Nam, D. H., Leifsson, P. S., \& Jensen, A. L. (2010). Mercury contamination in spotted seatrout, Cynoscion nebulosus: an assessment of liver, kidney, blood, and nervous system health. Science of the total environment, 408(23), 5808-5816

3. Bowerman IV, W. W., Evans, E. D., Giesy, J. P. \& Postupalsky, S. (1994). Using feathers to assess risk of mercury and selenium to bald eagle reproduction in the Great Lakes region. Archives of environmental contamination and toxicology, 27(3), 294-298.

4. Burger, J. 2013. Temporal trends (1989-2011) in levels of mercury and other heavy metals in feathers of fledgling great egrets nesting in Barnegat Bay, NJ. Environmental research.

5. Burger, J., Jehl Jr, J. R., y Gochfeld, M. (2013). Selenium: mercury molar ratio in eared grebes (Podiceps nigricollis) as a possible biomarker of exposure. Ecological Indicators, 34, 60-68.

6. Burger, J., y Gochfeld, M. (2004). Marine birds as sentinels of environmental pollution. EcoHealth, 1(3), 263-274.

7. Buroz, E. (1994). Métodos de evaluación de impactos II Curso de Postgrado sobre Evaluación de Impactos Ambientales. FLACAM. La Plata.

8. Cogua, P., Campos-Campos, N. H. \& Duque, G. (2012). Total mercury and methylmercury concentration in sediment and seston of Cartagena bay, colombian caribbean. Boletín de Investigaciones Marinas y Costeras-INVEMAR, 41(2), 267-285.

9. Corredor Rodríguez, C. (2013). Estado del arte sobre la presencia de mercurio en peces y su efecto en la salud. Tesis pregrado. Universidad Pontifica Javeriana. Sede Bogotá. Facultad de ciencias

10. Cristol, D. A., Mojica, E. K., Varian-Ramos, C. W. \& Watts, B. D. (2012). Molted feathers indicate low mercury in bald eagles of the Chesapeake Bay, USA. Ecological Indicators, 18, 20-24.

11. Cuentas, M. (2009). Evaluación cualitativa del impacto ambiental generado por la actividad minera en la rinconada Puno. (tesis de maestría). Universidad de Puira 
12. Gallarday, T. E. (2006). Estudio del impacto ambiental de la extracción aurífera artesanal dentro de la jurisdicción política del distrito de Santa Rosa de Quives, año 2006. Rev. Inst. investig. Fac. minas metal cienc. geogr, 9(18), 98-108.

13. Harris, H. H., Pickering, I. J. \& George, G. N. (2003). The chemical form of mercury in fish. Science, 301(5637), 1203-1203.

14. Lozada, J. \& Arends, E. (2000). Aspectos ambientales de los diferentes tipos de minería de oro, desarrollados en la Reserva Forestal Imataca. Revista Forestal Latinoamericana, 15(27), 81-99.

15. Marrugo, J. L., Ruiz, J. A. \& Díez, S. (2013). Relationship between mercury levels in hair and fish consumption in a population living near a hydroelectric tropical dam. Biological trace element research, 151(2), 187-194. DOI 10.1007/s12011-012-9561-z

16. Marrugo, J., Lans, E. \& Benítez, L. (2007). Finding of mercury in fish from the Ayapel marsh, Cordoba, Colombia. Revista MVZ Córdoba, 12(1), 878-886

17. Moreno Requena, J. A. (2013). Alteraciones comportamentales y de personalidad debido a la exposición ocupacional a mercurio en un grupo de mineros del oro de la región del Bagre Antioquia (Doctoral dissertation).
18. Ogola, J. S., Mitullah, W. V. \& Omulo, M. A. (2002). Impact of gold mining on the environment and human health: a case study in the Migori gold belt, Kenya. Environmental Geochemistry and Health, 24(2), 141-157.

19. Olivero, J., Johnson, B. \& Arguello, E. (2002). Human exposure to mercury in San Jorge river basin, Colombia (South America). Science of the total environment, 289(1), 41-47

20. Pegg, S. (2006). Mining and poverty reduction: Transforming rhetoric into reality. Journal of Cleaner Production, 14(3), 376-387.

21. Sousa, R. N., Veiga, M. M., Meech, J., Jokinen, J. \& Sousa, A. J. (2011). A simplified matrix of environmental impacts to support an intervention program in a small-scale mining site. Journal of Cleaner Production, 19(6), 580-587.

22. Swart, P. \& Dewulf, J. (2013). Quantifying the impacts of primary metal resource use in life cycle assessment based on recent mining data. Resources, Conservation and Recycling, 73, 180-187.

23. Urkidi, L. (2010). A glocal environmental movement against gold mining: Pascua-Lama in Chile. Ecological Economics, 70(2), 219-227.

24. USGS U.S. Geological Survey (2015). Recuperado de: https://www.usgs.gov/

\section{Conflicto de Intereses}

Los autores declaran no tener ningún conflicto de intereses 
UNAP 